\title{
Moisture-induced degradation of interfacial bond in FRP-strengthened
}

\section{masonry}

\author{
Hamid Maljaee ${ }^{1}$, Bahman Ghiassi ${ }^{2}$, Paulo B. Lourenço ${ }^{3}$, Daniel V. Oliveira ${ }^{4}$ \\ ISISE, University of Minho, Department of Civil Engineering, Guimarães, Portugal
}

\begin{abstract}
Externally bonded strengthening of masonry structures using Fiber Reinforced Polymers (FRPs) has been accepted as a promising technique. Although the effectiveness of FRPs in improving the performance of masonry components has been extensively investigated, their long-term performance and durability remain poorly addressed.

This paper, tackling one of the aspects related to durability of these systems, presents an experimental investigation on the effect of long-term (one year) water immersion on the performance of GFRP-strengthened bricks. The tests include materials' mechanical tests, as well as pull-off and single-lap shear bond tests, to investigate the changes in material properties and bond behavior with immersion time, respectively. The effect of mechanical surface treatment on the durability of the strengthened system as well as the reversibility of the degradation upon partial drying are also investigated. The experimental results are presented and critically discussed.
\end{abstract}

Keywords: FRP; Masonry; Bond; Moisture; Degradation.

${ }^{1}$ PhD Student, ISISE, University of Minho, Department of Civil Engineering, Azurém, 4800-058 Guimarães, Portugal. Phone: +351 253510 499, fax: +351 253510 217, E-mail: h.maljaee.civil@gmail.com

${ }^{2}$ Postdoctoral researcher, ISISE, University of Minho, Department of Civil Engineering, Azurém, 4800-058 Guimarães, Portugal. Phone: +351 253510 499, fax: +351 253510 217, E-mail: bahmanghiassi@civil.uminho.pt

${ }^{3}$ Professor, ISISE, University of Minho, Department of Civil Engineering, Azurém, 4800-058 Guimarães, Portugal. Phone: +351 253510 209, fax: +351253 510 217, E-mail: pbl@civil.uminho.pt

${ }^{4}$ Associate Professor, ISISE, University of Minho, Department of Civil Engineering, Azurém, 4800-058 Guimarães, Portugal. Phone: +351 253510 247, fax: +351 253510 217, E-mail: danvco@civil.uminho.pt 


\section{Introduction}

Modern composite materials such as Fiber Reinforced Polymers (FRP) have been increasingly used for Externally Bonded Reinforcement (EBR) of masonry structures due to their good mechanical properties, lightweight, and ease of application. FRPs are expected to have longer lifetime and lower maintenance requirements in comparison with conventional materials used for strengthening of civil structures [1]. The available information in the literature shows that EBR strengthening of masonry structures with FRP composites leads to a significant structural improvement, which is strongly correlated with the performance of the bond between the repair material and the substrate [2].

As the bond behavior is a key mechanism in the effectiveness of EBR systems [2-4], a clear understanding of its durability and long-term performance is critical for structural design and service life predictions [5-7]. The durability of bond in FRP-strengthened concrete elements has been extensively studied, see e.g. [8-10], while only few experimental studies can be found on masonry elements strengthened with FRP composites, see e.g. [5,11-13]. The bond behavior and its durability are affected by different factors including environmental conditions, composite material used, substrate properties, interfacial adhesion and the applied surface preparation. Environmental degradation agents that are likely to affect the performance of structural materials are moisture, alkalinity, temperature, creep, ultraviolet light and fire [1]. Moisture has been recognized as a critical agent that can reduce the bond shear strength, peak slip, integrity at the bond level and fracture energy in FRP-bonded elements $[5,14,15]$. The presence of water may cause unwelcomed changes at the level of matrix/substrate interface, as well as in fiber and polymer structures. Indeed, diffusion of water into organic polymers can result in chemical, mechanical and thermophysical changes. Water may create a weak boundary layer at the interface 
between composite material and the substrate. It is known that the interfacial fracture energy of the bonded region decreases due to moisture exposure $[15,16]$. In terms of chemical effects, water molecules can bind to the resin through hydrogen bonding and disrupt van der Waals forces inside the pore network, known as hydrolysis mechanism [17].

Despite the extensive information on the effect of water on the mechanical properties of epoxy resins and bonded joints, its effects on the bond in FRP-masonry systems have been rarely studied [5,11]. Sciolti et al. [11] investigated the effect of approximately six months water immersion on the bond performance in CFRP-strengthened calcareous stones and observed a 26\% reduction in the bond strength at the end of the tests. Ghiassi et al. [5] investigated the effect of six months water immersion on the bond behavior of GFRP-strengthened handmade clay bricks (with a relatively low compressive strength of $9.1 \mathrm{MPa}$ and no surface treatments) and observed a significant reduction of bond strength and fracture energy. However, in both cases, it was not clear if the degradation reached a plateau at the end of exposure or could further increase with exposure. The effect of surface treatment on durability of these systems as well as the reversibility of the observed degradation upon drying are also unknown. In addition, as masonry structures are made of rather a wide range of materials and unit shapes, investigating the durability of FRPstrengthened masonry systems considering different substrates is necessary.

This paper presents the effect of long-term (one-year) water immersion on the bond behavior and material properties in GFRP-strengthened (industrially produced) extruded bricks. The effect of mechanical surface treatment (grinding of the bricks' surfaces) on the durability of bond as well as reversibility of the observed degradation upon partial drying are also investigated. 


\section{Experimental program}

The experimental program consisted of one-year water immersion of GFRP-strengthened bricks and material constituents for investigating the water-induced mechanical degradation in the bond behavior and material properties. The specimens, after preparation and curing in the laboratory conditions, were immersed in water tanks specifically prepared for these tests. They were then periodically removed from the water (after different immersion periods) to perform the post-aging tests.

The post-ageing tests included weighing, visual inspection and mechanical characterization tests (materials tests and bond tests). The materials' mechanical tests included compressive tests on brick specimens and tensile tests on epoxy, primer and GFRP specimens. The bond characterization tests included pull-off and single-lap shear debonding tests.

The reversibility of the observed degradation was also investigated by testing (a) one set of specimens immediately after removal from water and (b) another set of specimens after one month storage in the laboratory conditions $\left(20^{\circ} \mathrm{C}\right.$ and $60 \%$ R.H $)$. The associated results are presented throughout the paper as "Wet" and "Partially dried" for the first and second group of specimens, respectively.

\subsection{Materials and specimens}

Materials consisted of (industrially produced) extruded solid clay bricks with dimensions of $200 \times 100 \times 50 \mathrm{~mm}^{3}$ as the substrate and GFRP sheets as the strengthening material. GFRP sheets were prepared following the wet lay-up procedure by impregnating a commercially available unidirectional E-glass fiber with a compatible two-part epoxy resin as the matrix. The epoxy resin 
was also used for adhesion of the GFRP sheets to the brick surface. A two-part epoxy primer was used for preparation of the bricks' surfaces before application of GFRP sheets.

The specimens prepared for material characterization tests included $40 \mathrm{~mm}$ cubic brick specimens, see Fig 1(a), dog-bone shaped epoxy and primer specimens, see Fig 1(b), and GFRP coupons, see Fig 1(c). The epoxy resin, primer and GFRP coupons were prepared according to the manufacturer's technical datasheets and ISO 527-1 [18] specifications. The specimens were cured for two months in the laboratory conditions before performing the tests as also followed in $[12,13]$. Two groups of specimens were prepared, following the wet lay-up procedure, for bond characterization tests. The first group was prepared without application of any mechanical surface treatment on the bricks' surfaces (denoted as ORG-specimens). These specimens were prepared for pull-off and debonding tests. The second group, consisting of only single-lap shear test specimens, was prepared using bricks with grinded surfaces (denoted as GR-specimens). In both sets of specimens, the bricks were initially cleaned and dried in an oven (for $24 \mathrm{hr}$ at $100^{\circ} \mathrm{C}$ ) followed by application of a primer layer on the brick's surfaces. The epoxy resin was then used for impregnation of the glass fibers and their application to the bricks' surfaces according to the geometrical details shown in Fig 2. The GFRP sheets were applied on the bricks' surfaces over a $180 \times 70 \mathrm{~mm}^{2}$ area for the pull-off test specimens, Fig 2(a). In case of the debonding test specimens, the GFRP sheets with $50 \mathrm{~mm}$ width were applied in a $150 \mathrm{~mm}$ length of the brick's surfaces leaving a $40 \mathrm{~mm}$ unbonded length at the loaded end, Fig 2(b).

\subsection{Water immersion}

The specimens, after two months of curing in the laboratory conditions, were immersed in water tanks for twelve months in a controlled temperature condition of $20^{\circ} \mathrm{C}$. The tanks were specifically used for these tests (i.e. only contained these specimens) and filled with pure water tap that was 
periodically changed and cleaned. According to the analysis reports from the water supplier, the water $\mathrm{pH}$ was in the range of 6.7-7.9 (with $\mathrm{SO}_{4}<10 \mathrm{mg} / \mathrm{L}, \mathrm{Mg}<1 \mathrm{mg} / \mathrm{L}, \mathrm{Cl}<10 \mu \mathrm{g} / \mathrm{L}$ and $\mathrm{Ca}=6$ $\mathrm{mg} / \mathrm{L}$ ) along the immersion period. However, as the water was changed periodically it is expected to have a similar condition along the investigated period. The specimens were taken periodically from the water bath, visually inspected and weighed. Materials' mechanical tests and bond tests (single-lap and pull-off) were then conducted on the specimens for quantitative evaluation of the degradation.

\subsection{Material characterization tests}

Material tests included compressive tests on cubic brick specimens and tensile tests on epoxy resin, primer and GFRP coupons. The test setup for each material characterization test is shown in Fig 3. Five specimens were tested for each material and testing type according to relevant test standards. The summary of the mechanical properties of the reference specimens is presented in Table 1.

The compressive tests were performed on $40 \mathrm{~mm}$ brick cubes according to the specifications provided by ASTM C67 [19] and EN 772-1 [20]. The tests were conducted with a Lloyds testing machine at the rate of $150 \mathrm{~N} / \mathrm{min}$ in the flatwise direction to obtain the compressive strength of the bricks.

The tensile tests on epoxy resin and primer were conducted according to ASTM D638 [21] to obtain the tensile strength and elastic modulus. The tests were carried out with a Lloyds testing machine at a displacement rate of $2 \mathrm{~mm} / \mathrm{min}$. Deformation of the specimens was monitored by a clip gauge placed on the middle of the specimens. The modulus of elasticity was obtained from the stress-strain curves as the slope of the chord between $20 \%$ and $60 \%$ of the maximum tensile stress. 
The tensile tests on GFRP coupons were performed according to ASTM D3039 [22] and ISO 5271 [18]. The tests were performed with an Instron testing machine at a displacement rate of $2 \mathrm{~mm} / \mathrm{min}$. The mechanical properties were obtained in terms of tensile strength and elastic modulus. As the specimens prepared following the wet lay-up procedure do not have a uniform thickness, fabric equivalent thickness was used for stress calculations according to ACI 440.3R [23] specifications. The elastic modulus was again obtained from the stress-strain curve as the slope of the chord between $20 \%$ and $60 \%$ of the maximum tensile stress.

\subsubsection{Bond tests}

For performing the pull-off tests, two $50 \mathrm{~mm}$ diameter partial cores (with a depth of around $5 \mathrm{~mm}$ ) were drilled on each specimen, see Fig 2(a). Thereafter, aluminum disks were glued over the GFRP surface by means of a high-strength epoxy paste adhesive. The specimens were placed on a rigid steel frame and firmly clamped to it to perform the tests. The pull-off tests were then carried out using a closed-loop servo-controlled testing machine by applying tensile forces to the aluminum disks with a displacement rate of $0.2 \mathrm{~mm} / \mathrm{min}$, see Fig 4(a). Two specimens were tested (resulting in four tests) in each immersion period.

The single-lab shear bond tests were performed using a closed-loop servo-controlled testing machine with maximum load capacity of $50 \mathrm{kN}$. A rigid supporting plate was used to support the specimens appropriately and to avoid any misalignments in the load application. The specimens were placed on the steel frame and firmly clamped to it as shown in Fig 4(b). The specimens were monotonically loaded in tension with a speed rate of $0.3 \mathrm{~mm} / \mathrm{min}$ under displacement control conditions. The specimens were instrumented with two LVDTs at the loaded end, one in the middle and one at the free end of the GFRP sheets to monitor the relative slip between GFRP composite and masonry substrate, see Fig 4(b). Three specimens were tested in each period. 


\section{Results and discussion}

\subsection{Visual inspection}

The specimens were visually inspected upon removal from the water bath to record any visible changes or interfacial delamination. A comparison between the photos taken before and after immersion presents a color change in the GFRP sheets in both groups of specimens (ORG- and GR-), see Fig 5. This can be resulted from oxidation of the ether and nitrogen groups in epoxy resin [24]. However, no interfacial delamination was observed in the aged specimens.

\subsection{Material properties}

The changes in the mechanical properties of materials are presented in this section as the mean value of five tested specimens.

The water absorption curve and the changes in the compressive strength of the bricks with water immersion are shown in Fig 6. A slight reduction of compressive strength can be observed in the specimens with a total reduction of about $12 \%$ after twelve months of immersion. As expected, the brick specimens became saturated in the first days of immersion (with $12.6 \%$ mass water absorption) and therefore the weight of the specimens did not change significantly throughout the tests, see Fig 6(a). The results also show that the degradation in the specimens is not reversed upon partial drying, although the brick specimens lost the whole absorbed water after one month drying in the laboratory conditions (around $0.3 \%$ mass water remained after one month laboratory storage).

The durability of bricks is generally related to the porosity, pore size distribution and vitrification degree [25]. The observed degradation can be due to chemical reactions of water with brick components $[5,25]$. Chemical reactions lead to formation of microcracks in clay bricks and thus 
reduction of mechanical properties. Ghiassi et al [5] reported a higher reduction of compressive strength (25\%) in handmade clay bricks, with compressive strength of 9.1, MPa after six months of water immersion. Handmade clay bricks generally have higher porosity and lower degree of vitrification and higher degradation rates are thus expected in them.

The average moisture absorption curves of epoxy resin, primer and GFRP specimens are shown in Fig 7. It seems that the epoxy resin has reached the saturation level after around eight months of water immersion with $2.5 \%$ mass water absorption. One month drying of the specimens in the laboratory conditions resulted in partial loss of the absorbed water (around $0.5 \%$ in each period). Primer had higher water absorption after twelve months of immersion (4.5\%) and also showed more water loss after partial drying. It also seems that the primer has not reached the saturation level at the end of immersion period. GFRP specimens seem to have reached the saturation level after about eight months of immersion with 1.9\% mass gain and around 50\% loss of absorbed water upon partial drying.

The variation of mechanical properties of "partially dried" epoxy resins is depicted in Fig 8. The tensile strength has decreased up to around $20 \%$ in the first five months and no more significant degradation can be observed, see Fig 8(a). Ghiassi et al [5] also reported a similar degradation in the tensile strength, $25 \%$, after six months of water immersion for a similar epoxy resin (corresponding to $1.2 \%$ of mass water absorption). The young's modulus, besides a slight increase, has not changed significantly, see Fig 8(b). An inverse trend, however, was observed and reported in $[5,26]$. The peak strain (strain corresponding to peak stress) has also decreased with water immersion resulting in a total $11 \%$ reduction after twelve months of immersion, see Fig 8(c).

The observed degradation in the epoxy resin can be due to several mechanisms. The foremost key in moist environment which needs to be taken into account is plasticization of adhesives [27]. The 
absorption of water results in plasticization, saponification or hydrolysis causing changes in the polymer's structure and thus mechanical properties [28]. Indeed, water molecules are capable of forming hydrogen bonds with epoxy molecules leading to swelling, dissolving, softening and mechanical degradation [29]. Lowering of the glass transition temperature $\left(T_{\mathrm{g}}\right)$ can also be observed which is usually associated with plasticization [29].

A comparison between the "partially dried" and "wet" epoxy resin specimens shows the mechanical strength of "partially dried" specimens is slightly enhanced only in the first months of immersion. This strength enhancement can probably be improved if the specimens are further dried.

The changes in mechanical properties of primer are shown in Fig 9. Regardless of fluctuations in the middle, $10 \%$ reduction was observed in tensile strength of primer during twelve months of water immersion. At the same period, the elastic modulus of primer decreased drastically up to $44 \%$, corresponding to a water uptake of around 4\%, see Fig 9. The primer specimens showed larger peak strains (strain corresponding to the peak stress) after immersion than the reference specimens (about $80 \%$ at the end), see Fig 9(c). The primer "wet" specimens were tested only until six months of immersion. Issues such as large variation of the obtained results in some exposure periods or experimental/technical problems led to the use of additional specimens in some immersion periods and thus insufficient available specimens to be tested in "wet" condition after six months. The results show a larger enhancement of mechanical properties upon partial drying in primer in comparison to epoxy resin. This can be due to the fact that the primer specimens lost larger amount of absorbed water upon drying, see Fig 7.

The variation of mechanical properties of GFRP coupons, tensile strength and elastic modulus, are illustrated in Fig 10. The maximum tensile strength was drastically dropped up to $27 \%$ after four 
months of water immersion followed by a slight degradation until twelve months (until $37 \%$ ). However, the elastic modulus was not changed significantly during the immersion. A relatively large coefficient of variation $(\mathrm{CoV})$ was seen in the tensile strength of GFRP coupons which is usual in wet lay-up manufactured specimens [5]. Ghiassi et al [5] reported a 40\% reduction of tensile strength and elastic modulus for a similar GFRP material after six months of water immersion. This difference is due to the lower water absorption of GFRP coupons in this study (around 2\% at the end) in comparison to the specimens prepared by Ghiassi et al [5] (around 3.5\% after six months). Here, a layer of epoxy resin was applied on the edges of GFRP coupons after cutting into appropriate sizes resulting in lower water absorption and thus lower degradation of mechanical properties. Looking at Fig 7(c), it can be observed that GFRP specimens lost approximately half of their weight after partial drying. Nevertheless, no reversibility was observed in the tensile strength and the elastic modulus.

Fig 10(c) shows a reduction in the peak strain with a similar trend as the changes of tensile strength in GFRP coupons. This was expected due to the linear behavior of GFRPs until failure and insignificant change of elastic modulus during the immersion period. The peak strain has reached a plateau after four months and a total reduction of $44 \%$ after twelve months. It has been reported that the water attack can result in corrosion of glass fibers and degradation of the fiber/epoxy interface $[29,30]$. Both of these degradation mechanisms can lead to reduction of mechanical properties in GFRP coupons and can be the main reasons of the observed degradation.

\subsection{Bond characterization}

\subsubsection{Pull-off test}

The variation of pull-off bond strength with immersion time together with typical failure mode is presented in Fig 11. As explained before, the pull-off tests were only performed on ORG- 
specimens. It can be observed that a severe degradation occurred during the first four months of water immersion. Thereafter, the pull-off strength reached a plateau with the total drop of $34 \%$ in twelve months of water immersion. It seems that partial drying of the specimens did not have any reversible effect on the bond strength. The typical failure mode in all of the specimens, including "wet" and "partially dried" ones, was cohesive failure with the fracture inside the brick. However, the thickness of the detached brick continuously decreased with increment of immersion period until reaching the primer impregnated depth, as also reported in [5].

\subsubsection{Single-lab shear test}

The envelop of the load-slip curves obtained from single-lab shear bond tests are presented in Fig 12 and Fig 13 for different periods of water immersion. A reduction of debonding force and bond stiffness (defined as the initial slope of the load-slip curves) can be observed with immersion time. This reduction is generally more in ORG-specimens which can be attributed to the lower adhesion quality in these specimens. Grinding the bricks' surfaces in GR-specimens results in removal of the top (weak and low porous) layer of the bricks, and thus improvement of the bond quality by better impregnation of the primer layer. It can be observed that the reference GRspecimens showed higher debonding force (23\%) and slip (36\%) than the reference ORGspecimens, see the reference curves in Fig 12 and Fig 13.

The load-slip curves corresponding to the middle LVDTs, see Fig 12 and Fig 13, show that the FRP slip is negligible in the middle, in comparison to the loaded end slip, until debonding initiates. Upon this point, the moment in which the load is at peak, the slip at the middle increases until complete debonding of the FRP. This is due to the fact that the effective bonded length is less than $50 \mathrm{~mm}$ (the distance between loaded end and middle LVDTs) in the (reference) specimens used in this study [31]. Effective bonded length is defined as the bonded length in which the 
stresses are transferred effectively to the substrate. A clear understanding of the changes of the effective bond length with time is of critical importance for design as it can lead to structure premature failure. However, this can only be made when strain gauges or full field measurement techniques are used in the tests. Therefore, a clear conclusion on the changes of the effective bond length cannot be made in this study.

The variation of the debonding force with immersion is illustrated in Fig 14 for both specimen types. A significant degradation is observed in ORG-specimens during the first six months of water immersion (around 33\%). Afterwards, no bond degradation is observed and it seems that the strength has reached a plateau. A relatively similar degradation (35\%) has also been reported in [5] for GFRP-strengthened bricks (handmade bricks with lower compressive strength) during six months of water immersion. A slight enhancement of the debonding force upon drying can be observed in the first months of immersion in ORG-specimens. However, this enhancement diminishes as the immersion period increases. In contrary to ORG-specimens, GR-specimens suffered only a slight reduction of $6 \%$ after 12 months of immersion. The results show that surface preparation has significantly affected the degradation level, as expected.

The typical failure modes of the specimens are presented in Fig 15. It can be seen that the failure in the reference specimens (both ORG- and GR-specimens) was cohesive with fracture inside a thin layer of the bricks. The water immersion resulted in change of failure mode in ORGspecimens to adhesive failure at the FRP/brick interface. However, no change of failure mode with water immersion was observed in GR-specimens. Nevertheless, it was observed that the thickness of remained detached brick on the GFRP sheet decreased during the exposure period.

According to the equation proposed by CNR DT200 [32], the bond fracture energy can be obtained from the debonding test results as follows: 
$G f=\frac{P_{\max }^{2}}{b_{f}^{2}\left(2 E_{f} t_{f}\right)}$

where $\mathrm{P}_{\max }$ is the debonding force, $b_{\mathrm{f}}$ is the FRP width, $E_{\mathrm{f}}$ is the FRP elastic modulus, and $t_{\mathrm{f}}$ is the FRP thickness. The $b_{\mathrm{f}}$ and $t_{\mathrm{f}}$ are assumed to be constant during the tests. Having the variation of the debonding force and FRP's elastic modulus, the changes of the bond fracture energy with water immersion can be obtained, see Fig 16. It seems that the fracture energy has not changed during the first two months of immersion. Afterwards, the degradation has initiated following an exponential trend until reaching a residual strength (after around six months of immersion) with $49 \%$ and $25 \%$ of reduction in ORG- and GR-specimens, respectively. The observed trend is very similar to degradation of bonded joints attacked by interfacial degradation and epoxy plasticization $[33,34]$. An exponential degradation model is used here for simulating the changes in the bond fracture energy, the solid line in Fig 16, assuming that the degradation only starts after two months of immersion.

The fracture energy can also be obtained, according to CNR DT200 [32], based on the mechanical properties of the substrate:

$G f=\frac{k_{b} \cdot k_{G}}{F C} \cdot \sqrt{f_{b m} \cdot f_{b t m}}$

where $k_{\mathrm{b}}, k_{\mathrm{G}}$ and $\mathrm{FC}$ are all constant factors, $f_{\mathrm{bm}}$ is the masonry compressive strength and $f_{\mathrm{btm}}$ is the masonry tensile strength, $k_{\mathrm{b}}$ is accounting for the width effects (can be obtained from CNR-DT200 [32]), $k_{\mathrm{G}}$ is a corrective factor proposed to be equal to $0.031 \mathrm{~mm}$ for FRP systems applied to bricks following the wet layup procedures, and FC is a design confidence factor which can be assumed equal to 1 (for the purpose of this study). The masonry tensile strength can be assumed equal to $0.1 \times f_{\mathrm{bm}}(10 \%$ of the masonry compressive strength). Thus the equation can be rewritten as:

$G f=k_{b} \cdot k_{G} \cdot \sqrt{0.1} f_{b m}$ 
Having the variation of masonry compressive strength from the experimental results and fracture energy from Eq. (1) and assuming that $k_{\mathrm{b}}$ is constant during the tests $\left(k_{\mathrm{b}}=1.29\right)$, the variation of parameter $k_{\mathrm{G}}$ with immersion time can be obtained from Eq. (3), see Fig 17. It should be noted that this relation is based on the assumption that the failure is cohesive inside the bricks and thus it is only applicable to GR-specimens. A linear regression analysis is performed on the obtained data leading to a time-dependent relation, presented in the figure, for this parameter. It can be observed that $k_{\mathrm{G}}$ has decreased about $10 \%$ after twelve months of immersion.

\section{Conclusions}

The effect of one year water immersion on the performance of GFRP-strengthened bricks (including the bond behavior and materials mechanical properties) was experimentally investigated in this paper. Industrially extruded solid clay bricks were used for the substrate as one of the common brick types used for masonry construction. The influence of mechanical surface treatment and partial drying of the specimens after immersion on the bond and material properties were also investigated. Partial drying was performed by one month storing the specimens in the laboratory conditions $\left(20^{\circ} \mathrm{C}\right.$ and $60 \%$ R.H.) before performing the post ageing tests.

The experimental results showed some degradation in the mechanical strength of bricks (12\%), epoxy resin (6\%), primer (10\%) and GFRP (37\%) after the immersion period. On the other hand, the elastic modulus did not significantly change in epoxy resin and GFRP but had a $44 \%$ drop in primer. The changes in the elastic modulus of bricks with water immersion were not

measured during the tests. In epoxy resin and primer specimens, a slight enhancement of mechanical properties was observed upon partial drying in the first months of water immersion. 
This can be due to the irreversibility of the induced degradation or insufficient drying of the specimens.

The changes in the bond performance were investigated by visual inspection, pull-off tests (only on specimens prepared with original bricks, ORG-specimens) and single-lap shear bond tests (on specimens prepared with original bricks, ORG-specimens, and grinded bricks, GR-specimens). The pull-off bond strength significantly decreased (34\%) after twelve months of immersion, but the failure mode remained cohesive with fracture inside the brick during all the immersion period. However, the thickness of the detached brick decreased with immersion time. The degradation seemed to only start after two months of immersion reaching a plateau after four months with an exponential trend.

The single-lap shear bond tests showed a significant improvement in durability of specimens prepared with grinded bricks (9\% reduction of bond strength in comparison to $19 \%$ in ORGspecimens), as expected. The failure mode of the GR-specimens remained cohesive with immersion time, with reduction of the thickness of the detached layer. On the other hand, the failure mode in ORG-specimens changed progressively from cohesive to adhesive failure mode. Again, partial drying of the specimens resulted in a slight enhancement of the bond strength in ORG-specimens in the first months of immersion. However, no strength enhancement was observed in GR-specimens.

Based on the observed experimental results, suitable degradation laws for bond fracture energy were extracted and presented. It was also observed that the parameter $k_{\mathrm{G}}$, in design formulas for obtaining the bond fracture energy, slightly (10\%) changes during the immersion period. 


\section{Acknowledgements}

The second author acknowledges the financial support of the Ministério da Ciência, Tecnologia e Ensino Superior, FCT, Portugal, under the grant SFRH/BPD/92614/2013.

\section{References}

[1] Karbhari VM, Chin JW, Hunston D, Benmokrane B, Juska T, Morgan R, et al. Durability Gap Analysis for Fiber-Reinforced Polymer Composites in Civil Infrastructure. J Compos Constr 2003;7:238-47. doi:10.1061/(ASCE)1090-0268(2003)7:3(238).

[2] Aiello MA, Sciolti SM. Bond analysis of masonry structures strengthened with CFRP sheets. Constr Build Mater 2006;20:90-100. doi:10.1016/j.conbuildmat.2005.06.040.

[3] Oliveira D V., Basilio I, Lourenço PB. Experimental Bond Behavior of FRP Sheets Glued on Brick Masonry. J Compos Constr 2011;15:32-41. doi:10.1061/(ASCE)CC.19435614.0000147.

[4] Ghiassi B, Verstrynge E, Lourenço PB, Oliveira D V. Characterization of debonding in FRP-strengthened masonry using the acoustic emission technique. Eng Struct 2014;66:2434. doi:10.1016/j.engstruct.2014.01.050.

[5] Ghiassi B, Marcari G, Oliveira D V., Lourenço PB. Water degrading effects on the bond behavior in FRP-strengthened masonry. Compos Part B Eng 2013;54:11-9.

doi:10.1016/j.compositesb.2013.04.074.

[6] Ouyang Z, Wan B. Experimental and Numerical Study of Moisture Effects on the Bond Fracture Energy of FRP/Concrete Joints. J Reinf Plast Compos 2007;27:205-23.

doi:10.1177/0731684407082952.

[7] Böer P, Holliday L, Kang THK. Interaction of environmental factors on fiber-reinforced polymer composites and their inspection and maintenance: A review. Constr Build Mater 2014;50:209-18. doi:10.1016/j.conbuildmat.2013.09.049.

[8] Silva MAG, Biscaia H. Degradation of bond between FRP and RC beams. Compos Struct 2008;85:164-74. doi:10.1016/j.compstruct.2007.10.014.

[9] Tuakta C, Büyüköztürk O. Conceptual Model for Prediction of FRP-Concrete Bond Strength under Moisture Cycles. J Compos Constr 2011;15:743-56. doi:10.1061/(ASCE)CC.1943-5614.0000210. 
[10] Karbhari VM, Ghosh K. Comparative durability evaluation of ambient temperature cured externally bonded CFRP and GFRP composite systems for repair of bridges. Compos Part A Appl Sci Manuf 2009;40:1353-63. doi:10.1016/j.compositesa.2009.01.011.

[11] Sciolti MS, Aiello MA, Frigione M. Influence of water on bond behavior between CFRP sheet and natural calcareous stones. Compos Part B Eng 2012;43:3239-50. doi:10.1016/j.compositesb.2012.03.002.

[12] Ghiassi B, Lourenço PB, Oliveira D V. Accelerated Hygrothermal Aging of Bond in FRP-Masonry Systems. J Compos Constr 2014:04014051. doi:10.1061/(ASCE)CC.19435614.0000506.

[13] Ghiassi B, Oliveira D V, Lourenco PB. Hygrothermal durability of bond in FRPstrengthened masonry. Mater Struct 2014;47:2039-50. doi:10.1617/s11527-014-0375-7.

[14] Lau D, Büyüköztürk O. Fracture characterization of concrete/epoxy interface affected by moisture. Mech Mater 2010;42:1031-42. doi:10.1016/j.mechmat.2010.09.001.

[15] Nguyen T, Byrd E, Alsheh D, Apuadi K, Chin J. Water at the polymer/substrate interface and its role in the durability of polymer/glass fibre composites. In: Benmokrane, B.; Rahman H, editor. CDCC'98, Sherbrooke Canada: n.d., p. 451-62.

[16] Kinloch AJ. The Science of Adhesion, Part 1: Surface and Interfacial Aspects. J Mater Sci 1980;15:2141-66.

[17] Zhou J, Lucas JP. Hygrothermal effects of epoxy resin. Part I: the nature of water in epoxy. Polymer (Guildf) 1999;40:5505-12. doi:10.1016/S0032-3861(98)00790-3.

[18] ISO 527-1. Plastics -Determination of tensile properties -Part 1: General principles. 2012.

[19] ASTM C67. Standard Test Methods for Sampling and Testing Brick and Structural Clay Tile. vol. 04. 2014. doi:10.1520/C0067-12.2.

[20] EN 772-1. Methods of test for masonry units. Determination of compressive strength. 2011.

[21] ASTM D638. Standard Test Method for Tensile Properties of Plastics 1. 2010.

[22] ASTM D3039/D 3039M. Standard Test Method for Tensile Properties of Polymer Matrix Composite Materials 1. 2014.

[23] ACI 440.3R. Guide test methods for Fibre-Reinforced Polymers (FRPs) for reinforcing or strengthening concrete structures. 2004.

[24] Stewart A. Study of cement - epoxy interfaces, accelerated testing, and surface modification. University of Florida, 2012. 
[25] Elert K, Cultrone G, Rodriguez Navarro C, Sebastián Pardo E. Durability of bricks used in the conservation of historic buildings - Influence of composition and microstructure. $\mathrm{J}$ Cult Herit 2003;4:91-9. doi:10.1016/S1296-2074(03)00020-7.

[26] Tuakta C, Büyüköztürk O. Deterioration of FRP/concrete bond system under variable moisture conditions quantified by fracture mechanics. Compos Part B Eng 2011;42:14554. doi:10.1016/j.compositesb.2010.11.002.

[27] Brewis DM, Comyn J, Shalash RJA. The effect of moisture and temperature on the properties of an epoxide-polyamide adhesive in relation to its performance in single lap joints. Adhes Adhes 1982;2:215-22.

[28] Hollaway LC. A review of the present and future utilisation of FRP composites in the civil infrastructure with reference to their important in-service properties. Constr Build Mater 2010;24:2419-45. doi:10.1016/j.conbuildmat.2010.04.062.

[29] Hutchinson AR, Hollaway LC. Environmental durability. Strength. Reinf. Struct. Using externally-bonded FRP Compos. Struct. Civ. Eng., Cambridge: Woodhead Publishing Ltd; 2001, p. 156-82.

[30] Schutte CL. Environmental durability of glass-fiber composites. Mater Sci Eng R Reports 1994; 13:265-323.

[31] Ghiassi B, Xavier J, Oliveira D V., Lourenço PB. Application of digital image correlation in investigating the bond between FRP and masonry. Compos Struct 2013;106:340-9. doi:10.1016/j.compstruct.2013.06.024.

[32] CNR-DT 200 R1. Guide for the Design and Construction of Externally Bonded FRP Systems for Strengthening Existing Structures. 2013.

[33] Bowditch MR. The durability of adhesive joints in the presence of water. Int J Adhes Adhes 1996;16:73-9. doi:10.1016/0143-7496(96)00001-2.

[34] Ghiassi B. Durability analysis of bond between composite materials and masonry substrates. University of Minho, 2013. 


\section{List of Tables}

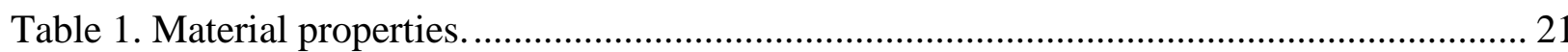

\section{List of Figures}

Fig 1. Geometry of specimens prepared for material characterization: (a) brick cubes; (b) epoxy resin and primer; (c) GFRP coupon (dimensions are in $\mathrm{mm}$ ).

Fig 2. Geometrical details of the specimens for (a) pull-off tests; (b) debonding tests (dimensions are in $\mathrm{mm}$ ).

Fig 3. Mechanical tests setup: (a) brick compressive test; (b) epoxy and primer tensile test; (c) GFRP tensile test.

Fig 4. Bond tests setup: (a) pull-off test; (b) single-lap shear tests.

Fig 5. Typical photos of specimens before and after water immersion.

Fig 6. (a) Moisture absorption curve of cubic brick; (b) Brick's compressive strength with immersion time.

Fig 7. Moisture absorption curves of: (a) epoxy resin; (b) primer; (c) GFRP coupons.

Fig 8. Tensile properties of epoxy resin with water immersion: (a) tensile strength; (b) elastic modulus; (c) peak strain.

Fig 9. Tensile properties of primer with water immersion: (a) tensile strength; (b) elastic modulus; (c) peak strain.

Fig 10. Tensile properties of GFRP coupons with water immersion: (a) tensile strength; (b) elastic modulus; (c) peak strain.

Fig 11. Pull-off strength with immersion time.

Fig 12. Force-slip curves of "partially dried" ORG-specimens with water immersion.

Fig 13. Force-slip curves of "partially dried" GR-specimens with water immersion.

Fig 14. Debonding force with immersion time.

Fig 15. Failure mode changes with water immersion.

Fig 16. Bond fracture energy with immersion time in (a) ORG-specimens; (b) GR-specimens.

Fig 17. Changes of parameter $k_{\mathrm{G}}$ with immersion time. 
Table 1. Material properties.

\begin{tabular}{ccccc}
\hline & $\begin{array}{c}\text { Compressive } \\
\text { strength } \\
(\mathrm{MPa})\end{array}$ & $\begin{array}{c}\text { Tensile strength } \\
(\mathrm{MPa})\end{array}$ & $\begin{array}{c}\text { Elastic } \\
\text { modulus } \\
(\mathrm{GPa})\end{array}$ & $\begin{array}{c}\text { Strain at peak } \\
\text { load } \\
(\%)\end{array}$ \\
\hline Brick & $16.76(\mathrm{COV}: 10.9 \%)$ & - & - & - \\
Epoxy resin & - & $44.6(\mathrm{COV}: 11.7 \%)$ & $\begin{array}{c}2.17(\mathrm{COV}: \\
6.5 \%)\end{array}$ & $2.12(\mathrm{COV}: 16.0 \%)$ \\
Primer & - & $38.7(\mathrm{COV}: 19.8 \%)$ & $\begin{array}{c}2.48(\mathrm{COV}: \\
3.8 \%)\end{array}$ & $1.64(\mathrm{COV}: 24.2 \%)$ \\
GFRP & - & $1087(\mathrm{COV}: 17.6 \%)$ & $58.2(\mathrm{COV}: 3.9 \%$ & $1.95(\mathrm{COV}: 11.7 \%)$ \\
coupons & - & & &
\end{tabular}




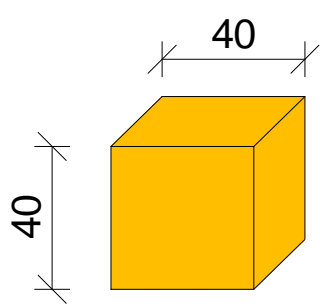

(a)

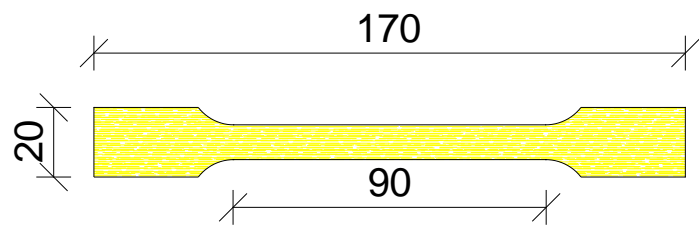

(b)

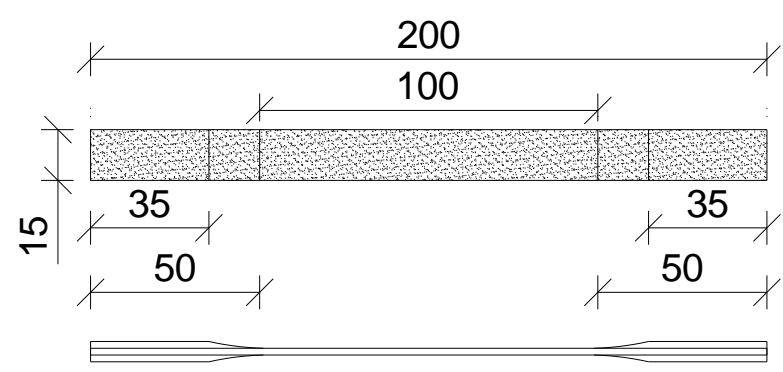

(c)

Fig 1. Geometry of specimens prepared for material characterization: (a) brick cubes; (b) epoxy resin and primer; (c) GFRP coupon (dimensions are in $\mathrm{mm}$ ). 
(a)

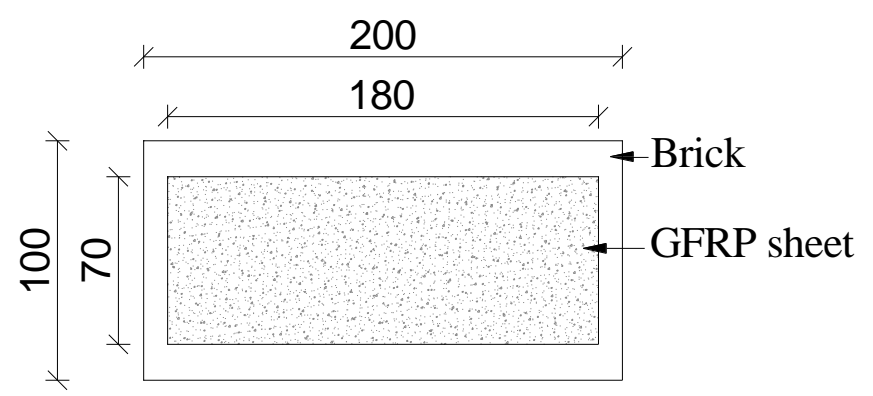

(b)

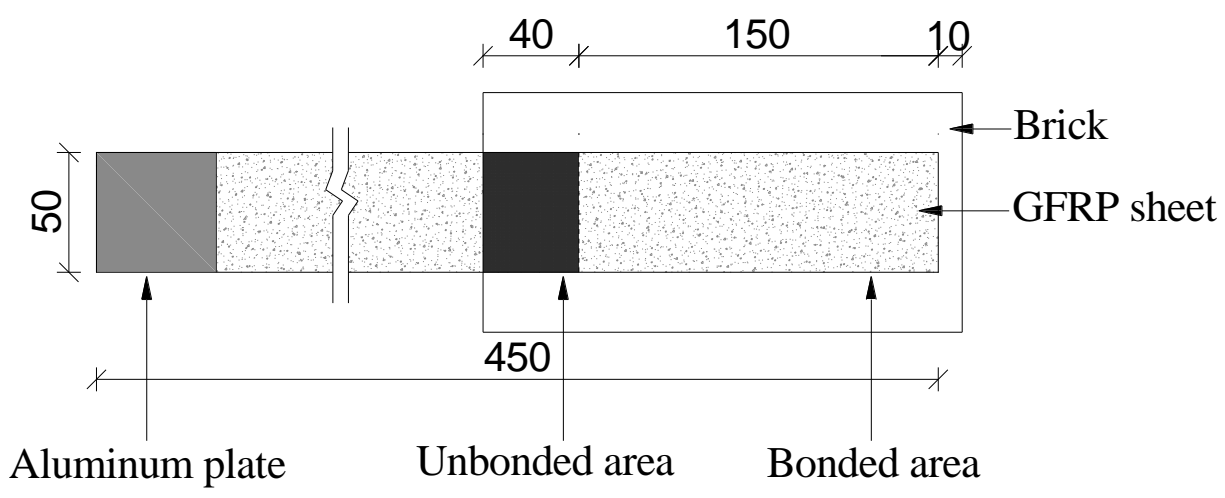

Fig 2. Geometrical details of the specimens for (a) pull-off tests; (b) debonding tests (dimensions are in $\mathrm{mm}$ ). 
(a)

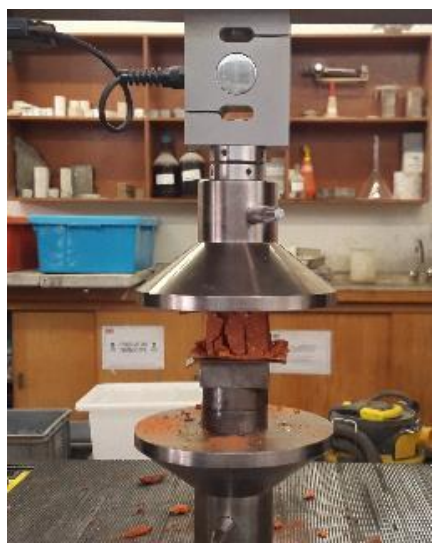

(b)

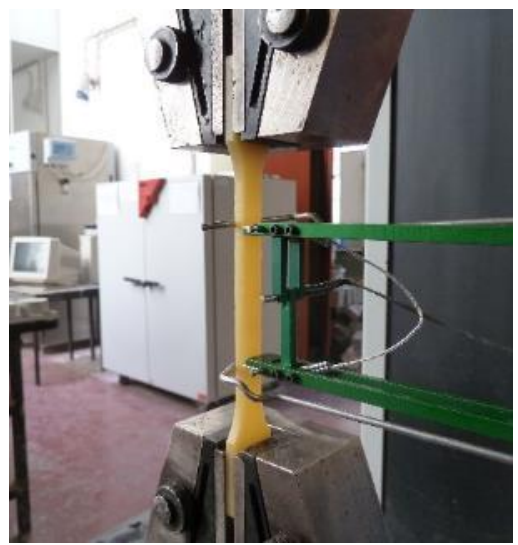

(c)

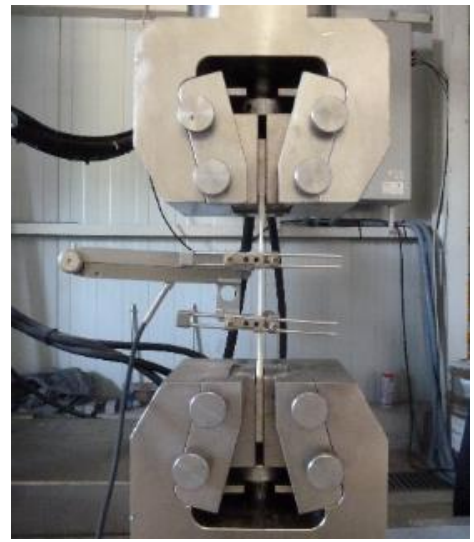

Fig 3. Mechanical tests setup: (a) brick compressive test; (b) epoxy and primer tensile test; (c) GFRP tensile test. 
(a)
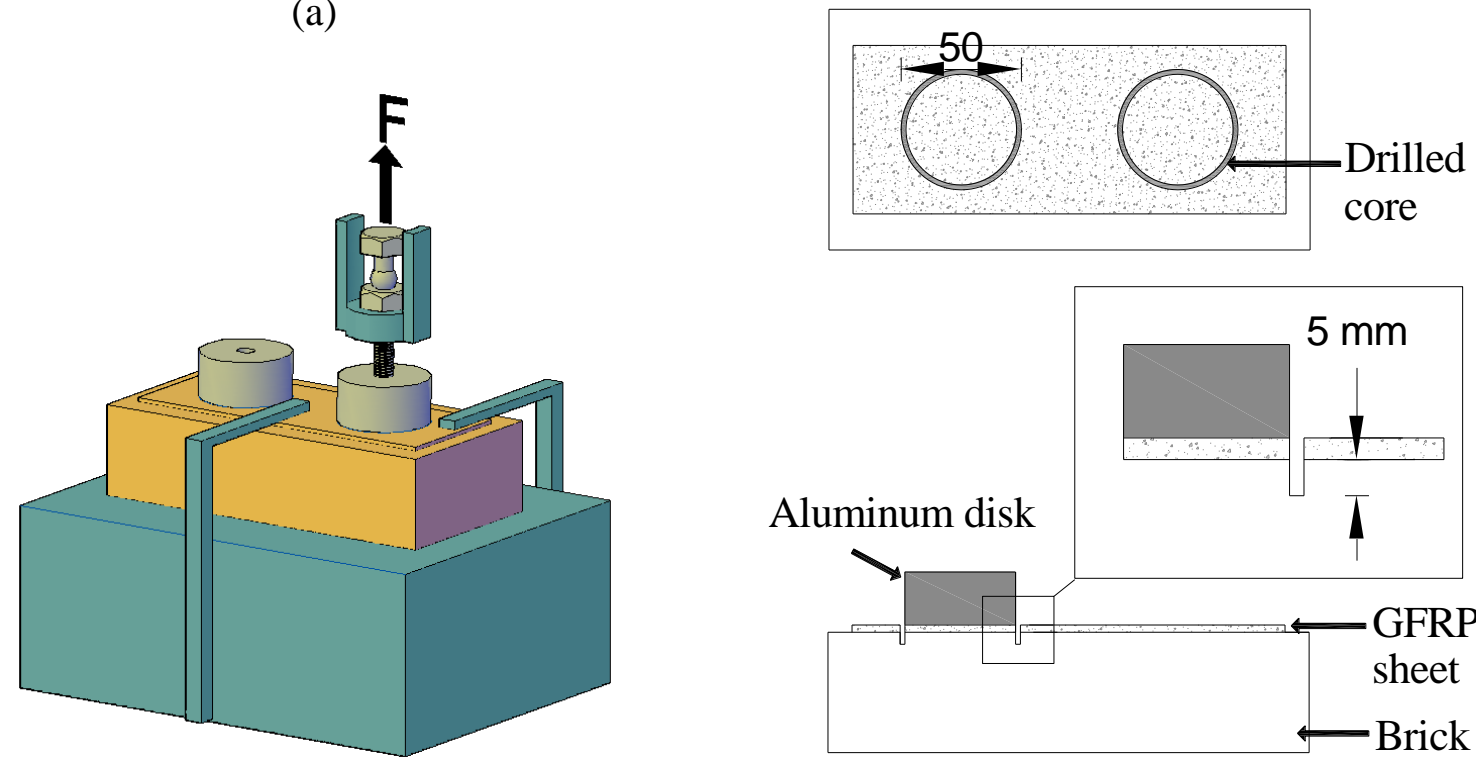

(b)
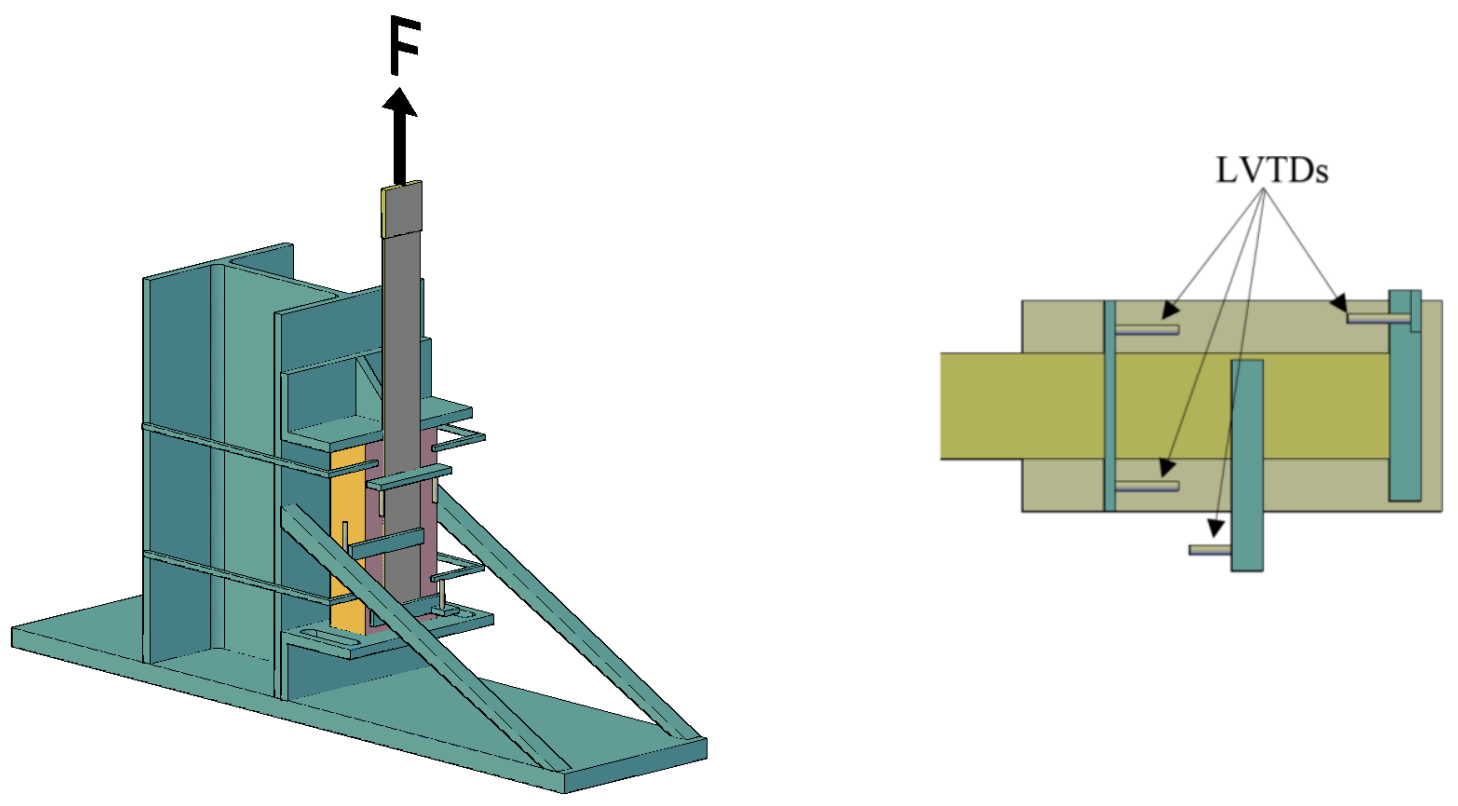

Fig 4. Bond tests setup: (a) pull-off test; (b) single-lap shear tests. 
ORG-specimens

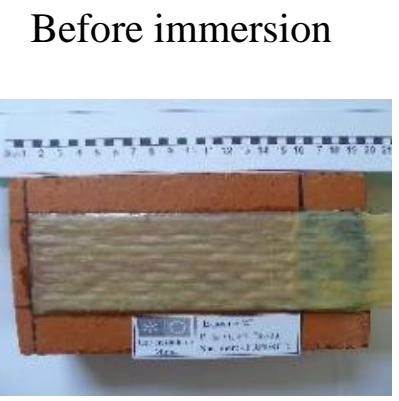

GR-specimens

After immersion

Before immersion

After immersion
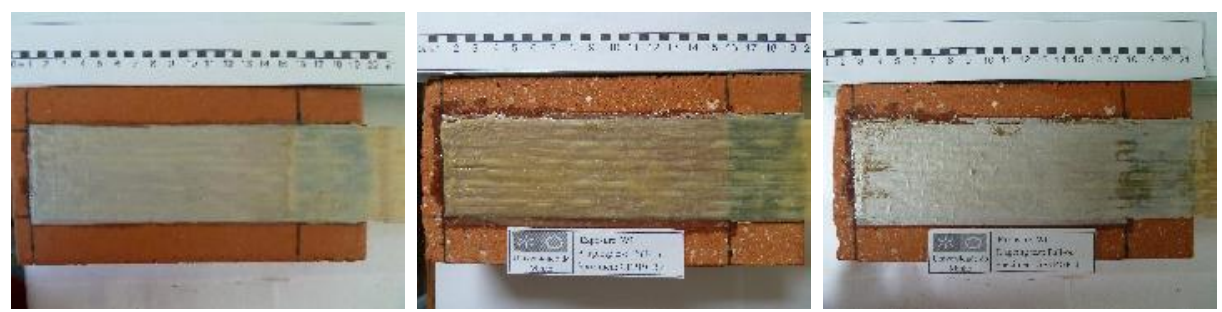

Fig 5. Typical photos of specimens before and after water immersion. 
(a)

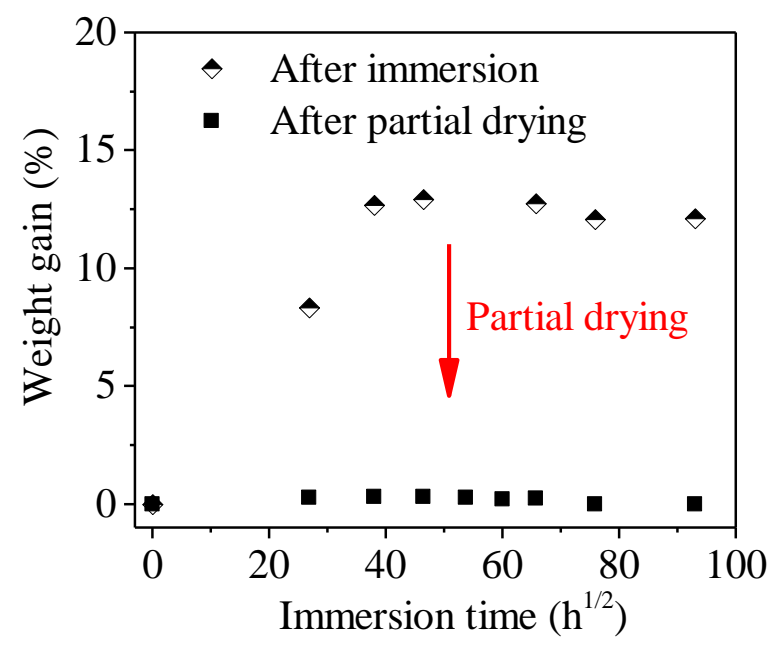

(b)

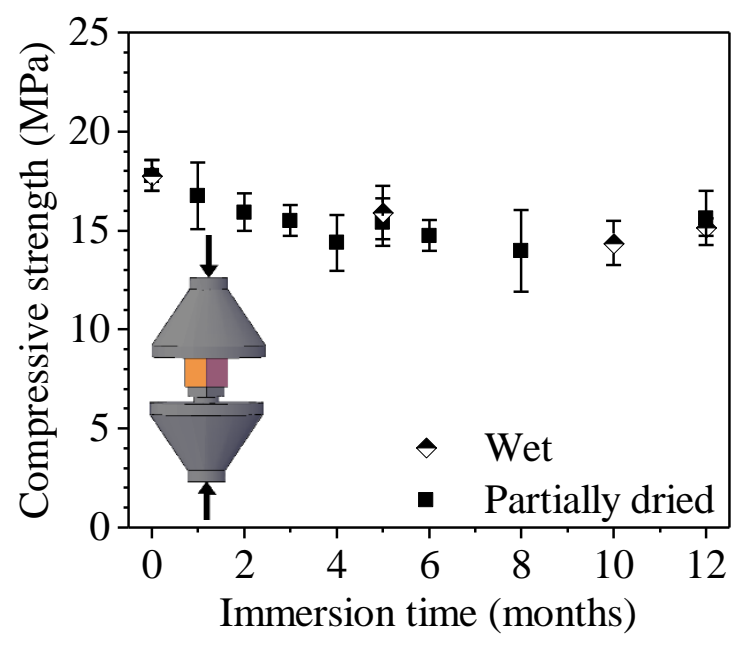

Fig 6. (a) Moisture absorption curve of cubic brick; (b) Brick's compressive strength with immersion time. 
(a)

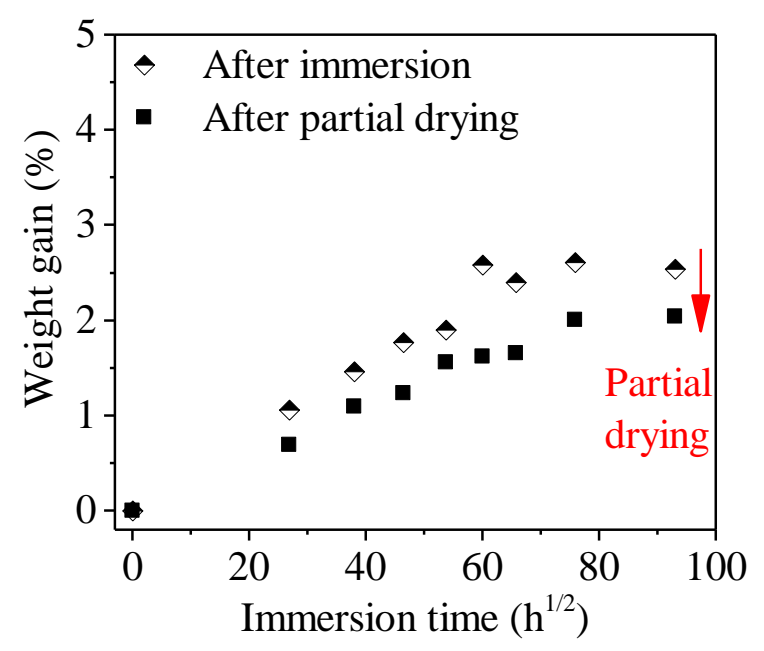

(b)

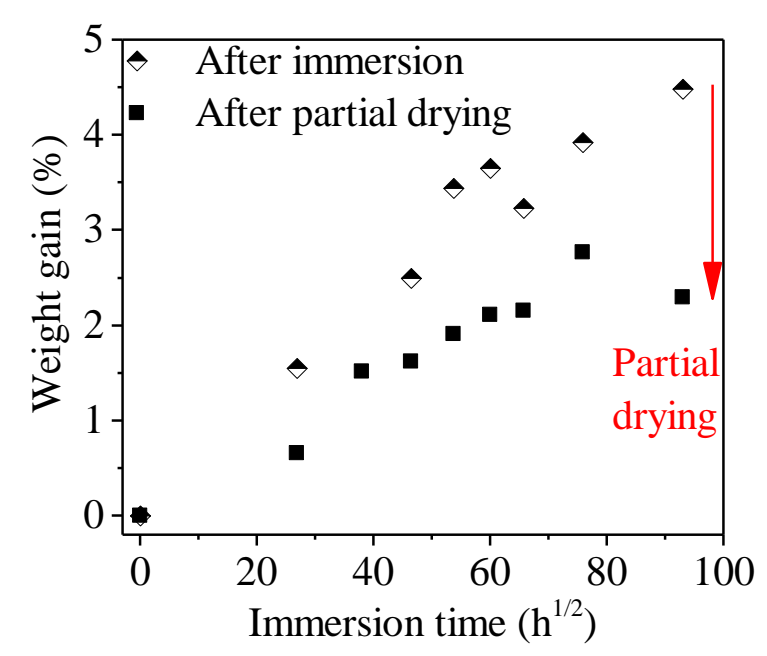

(c)

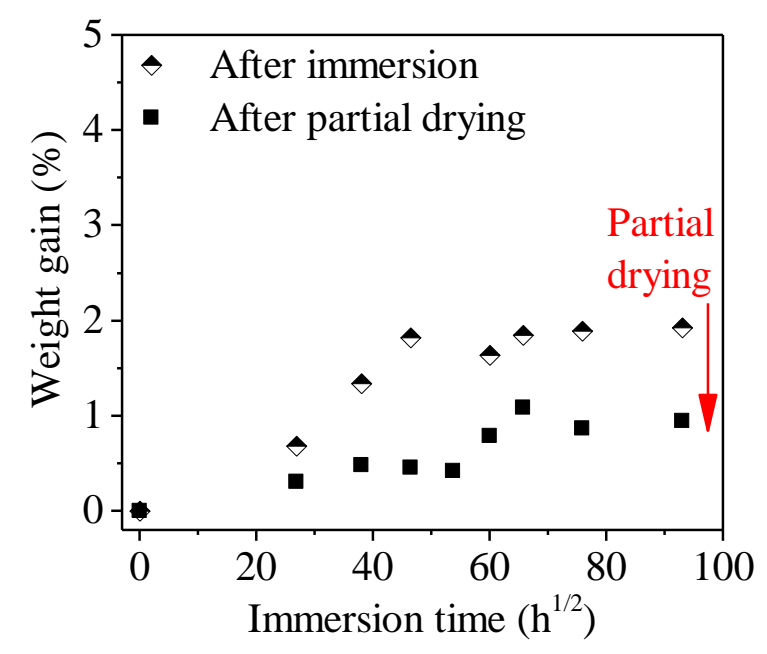

Fig 7. Moisture absorption curves of: (a) epoxy resin; (b) primer; (c) GFRP coupons. 
(a)

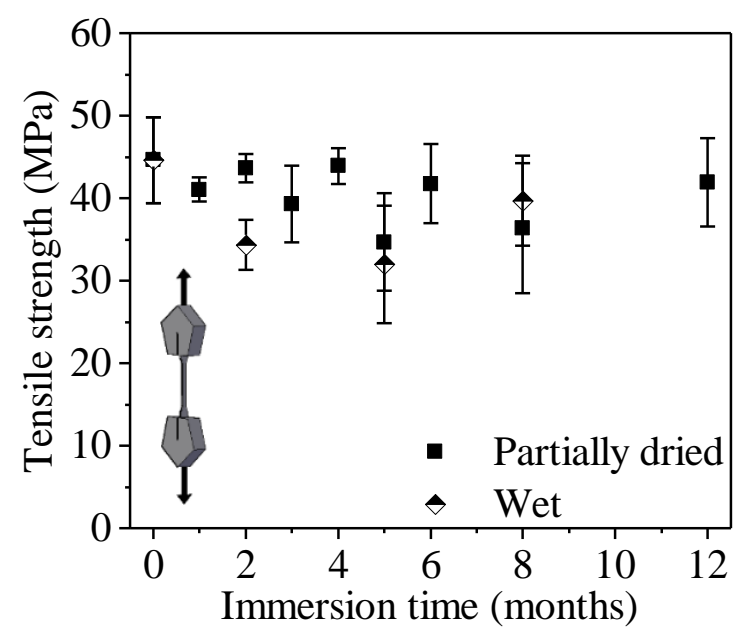

(b)

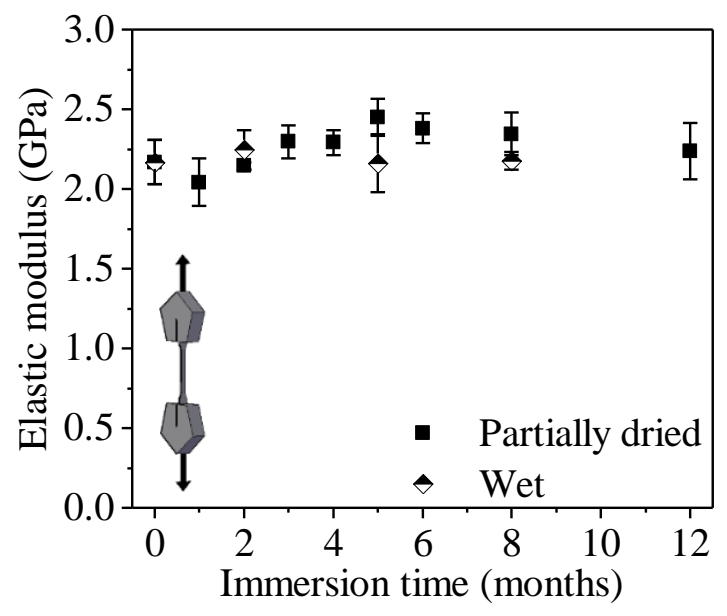

(c)

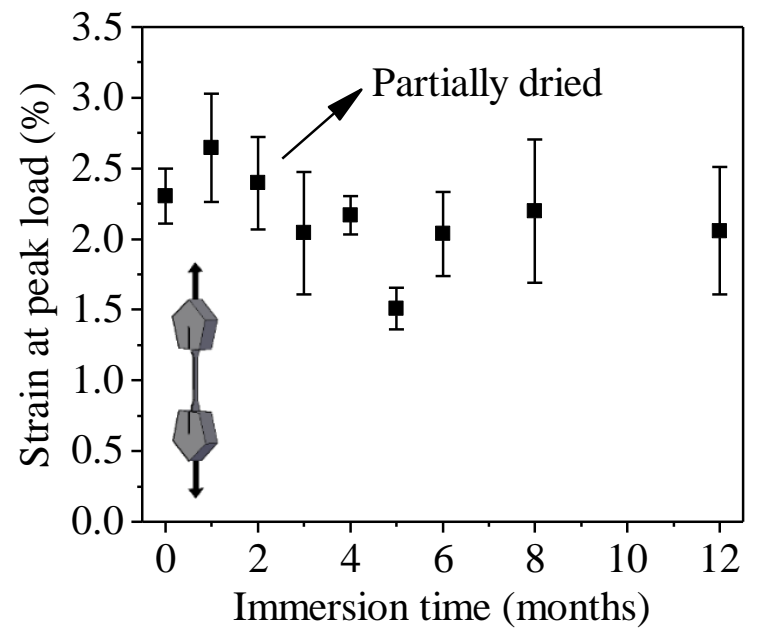

Fig 8. Tensile properties of epoxy resin with water immersion: (a) tensile strength; (b) elastic modulus; (c) peak strain. 
(a)

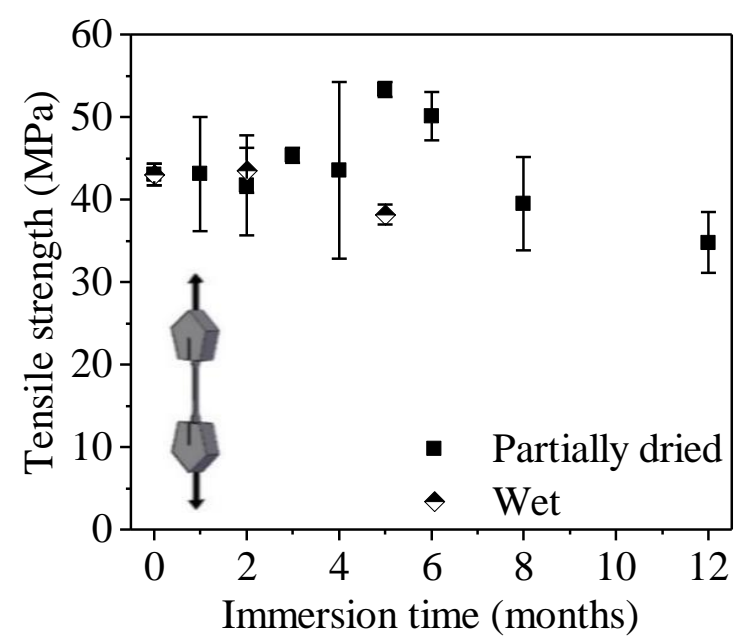

(b)

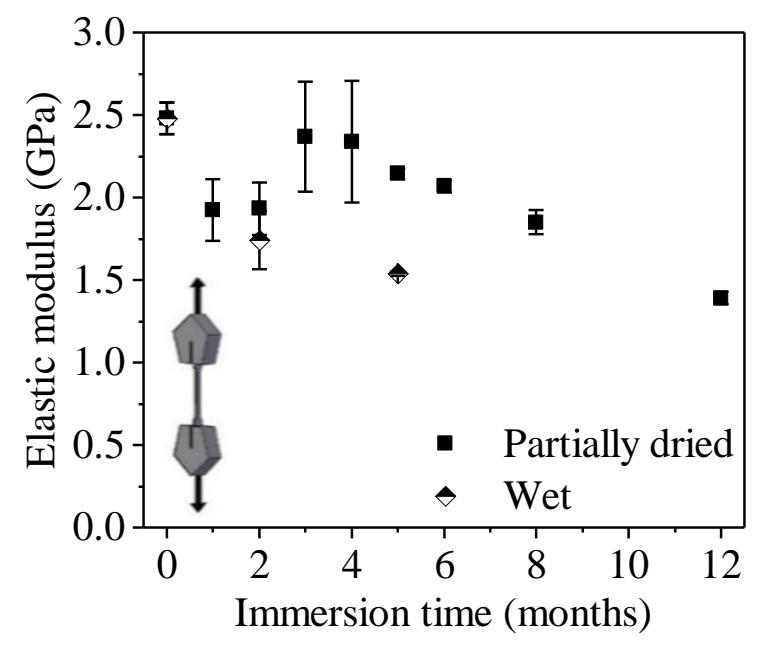

(c)

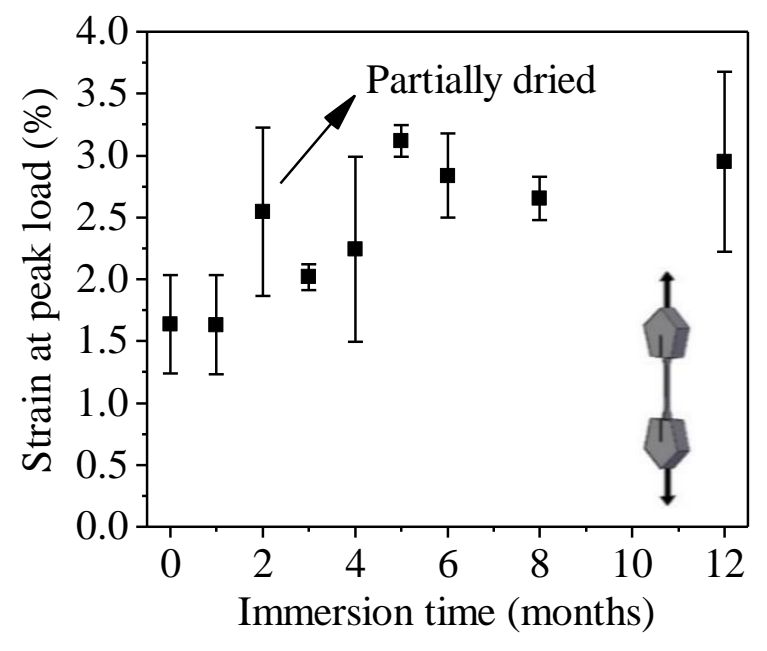

Fig 9. Tensile properties of primer with water immersion: (a) tensile strength; (b) elastic modulus; (c) peak strain. 
(a)

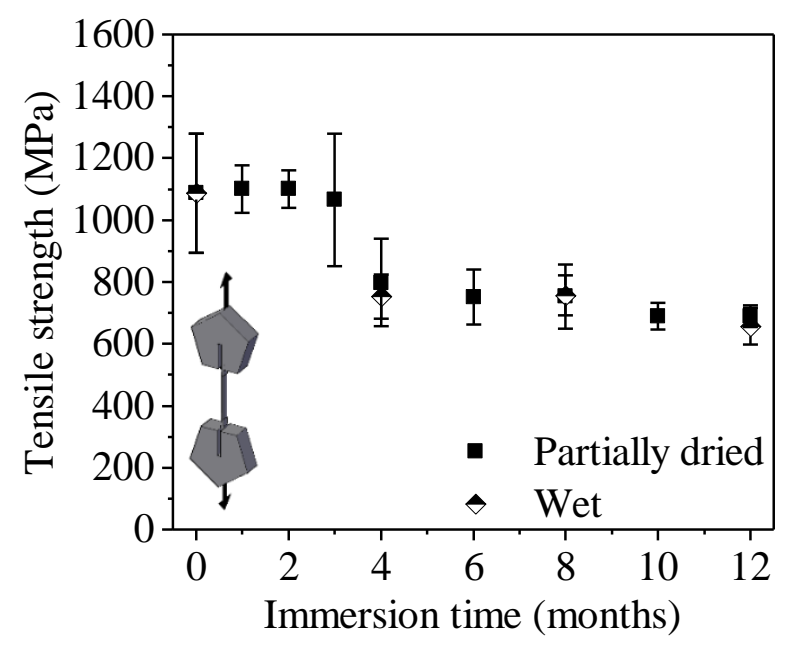

(b)

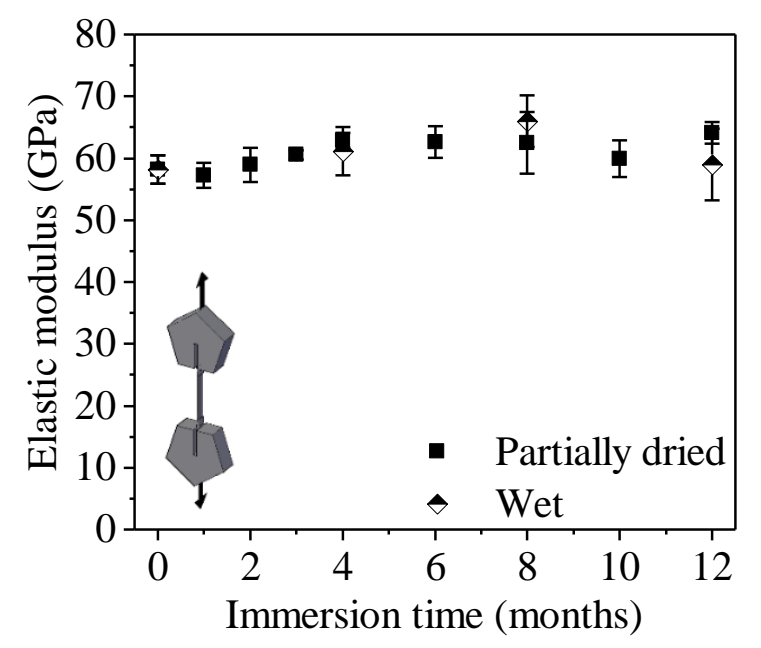

(c)

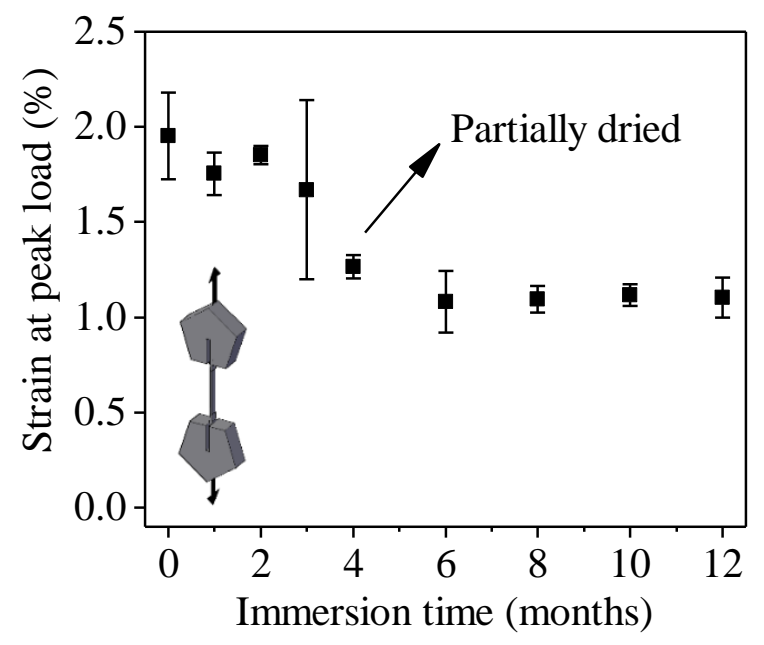

Fig 10. Tensile properties of GFRP coupons with water immersion: (a) tensile strength; (b) elastic modulus; (c) peak strain. 


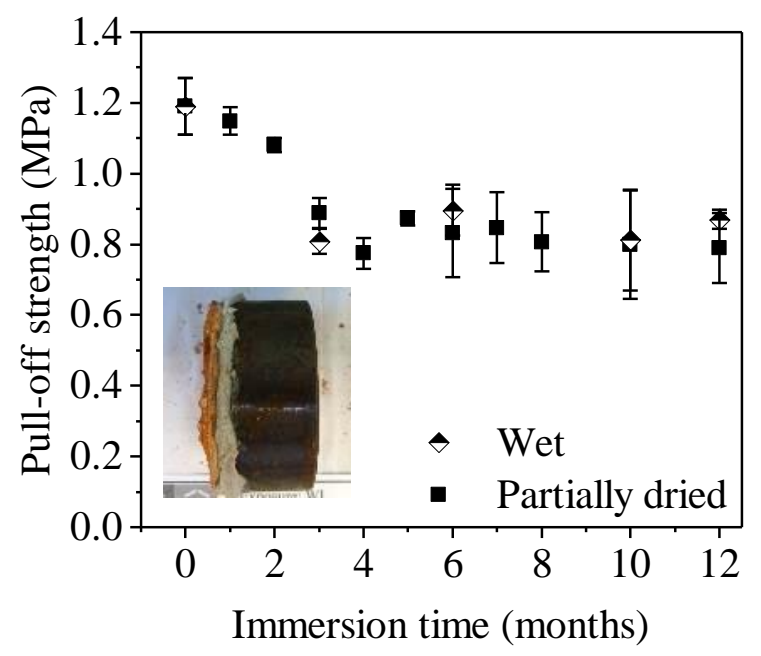

Fig 11. Pull-off strength with immersion time. 
Reference

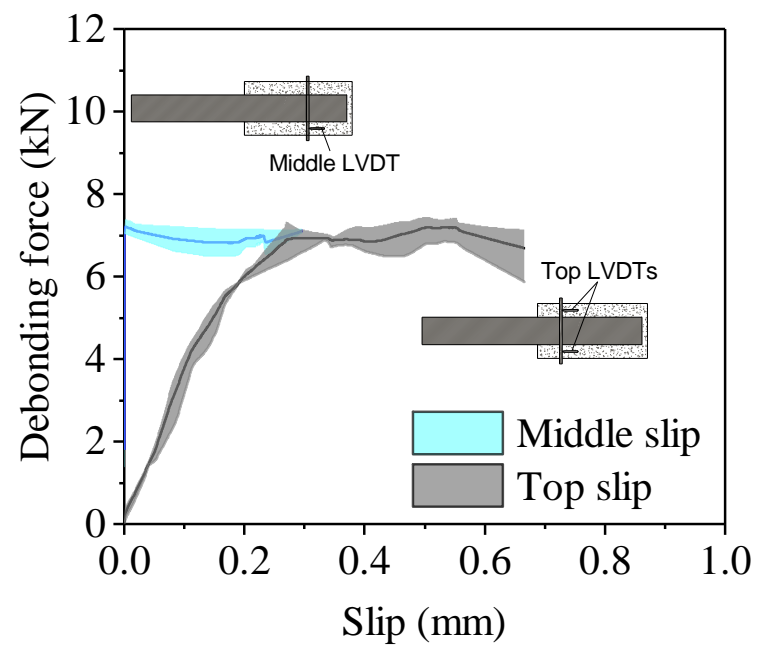

8 months of water immersion

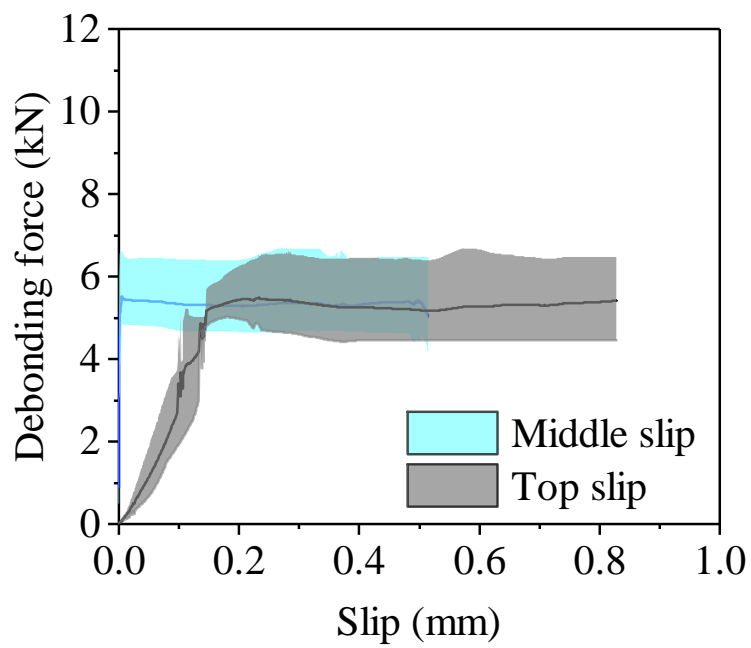

12 months of water immersion

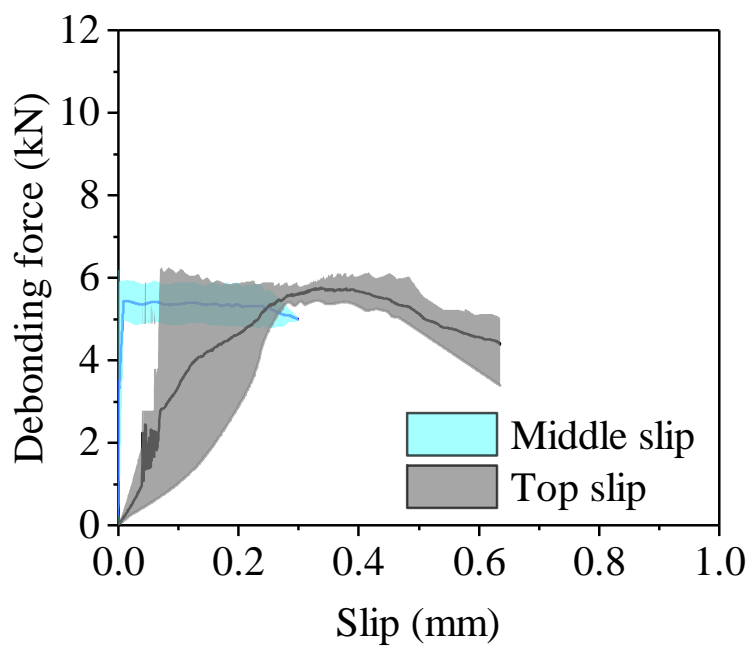

Fig 12. Force-slip curves of "partially dried" ORG-specimens with water immersion. 
Reference

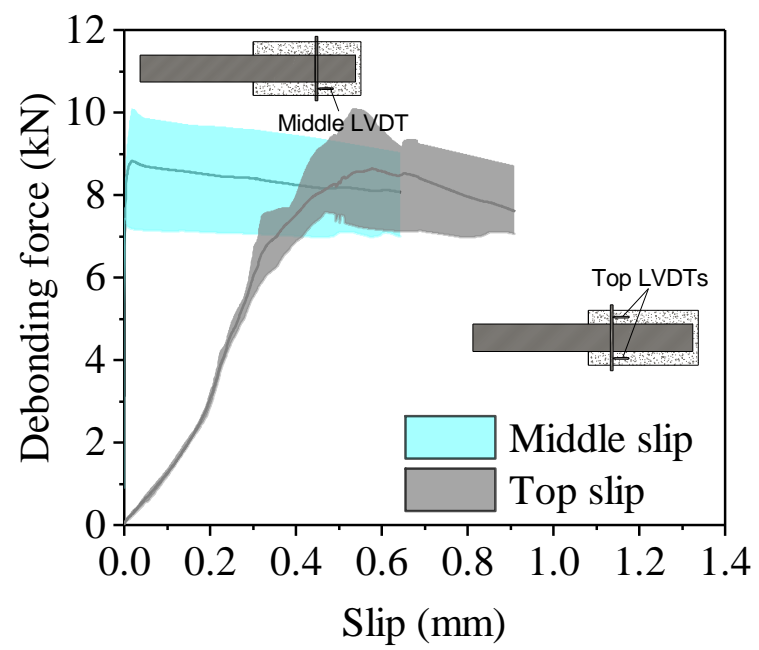

8 months of water immersion

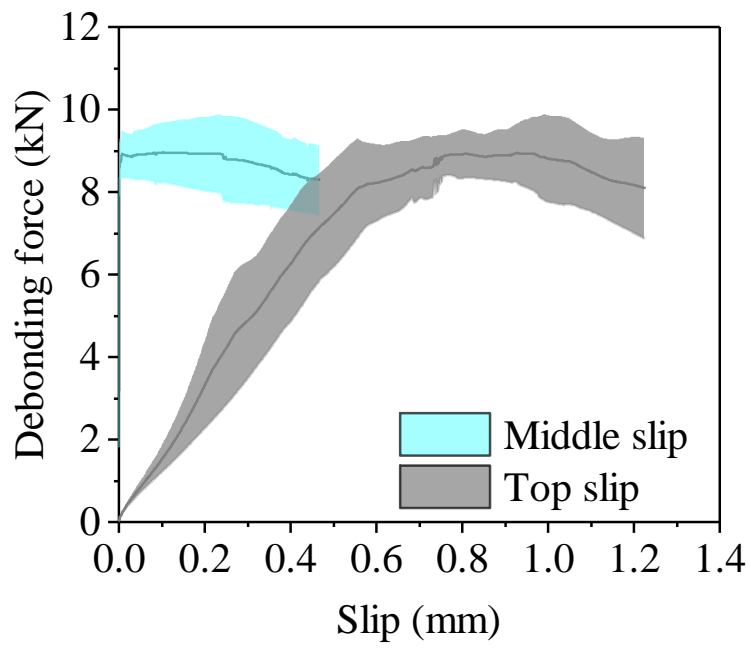

12 months of water immersion

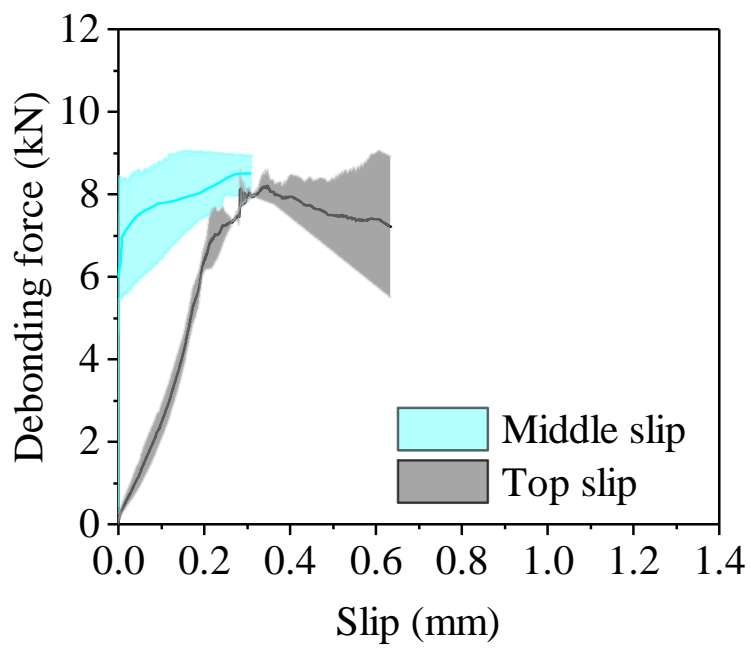

Fig 13. Force-slip curves of "partially dried" GR-specimens with water immersion. 


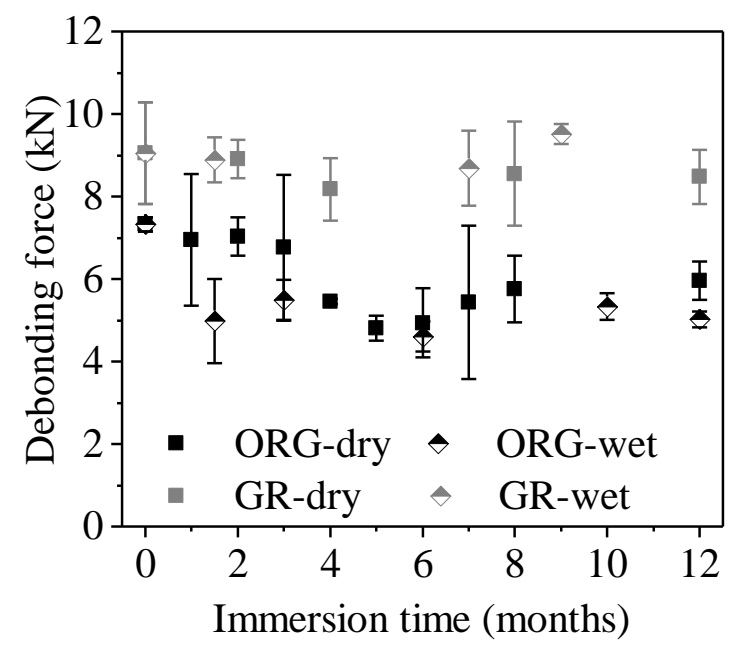

Fig 14. Debonding force with immersion time. 


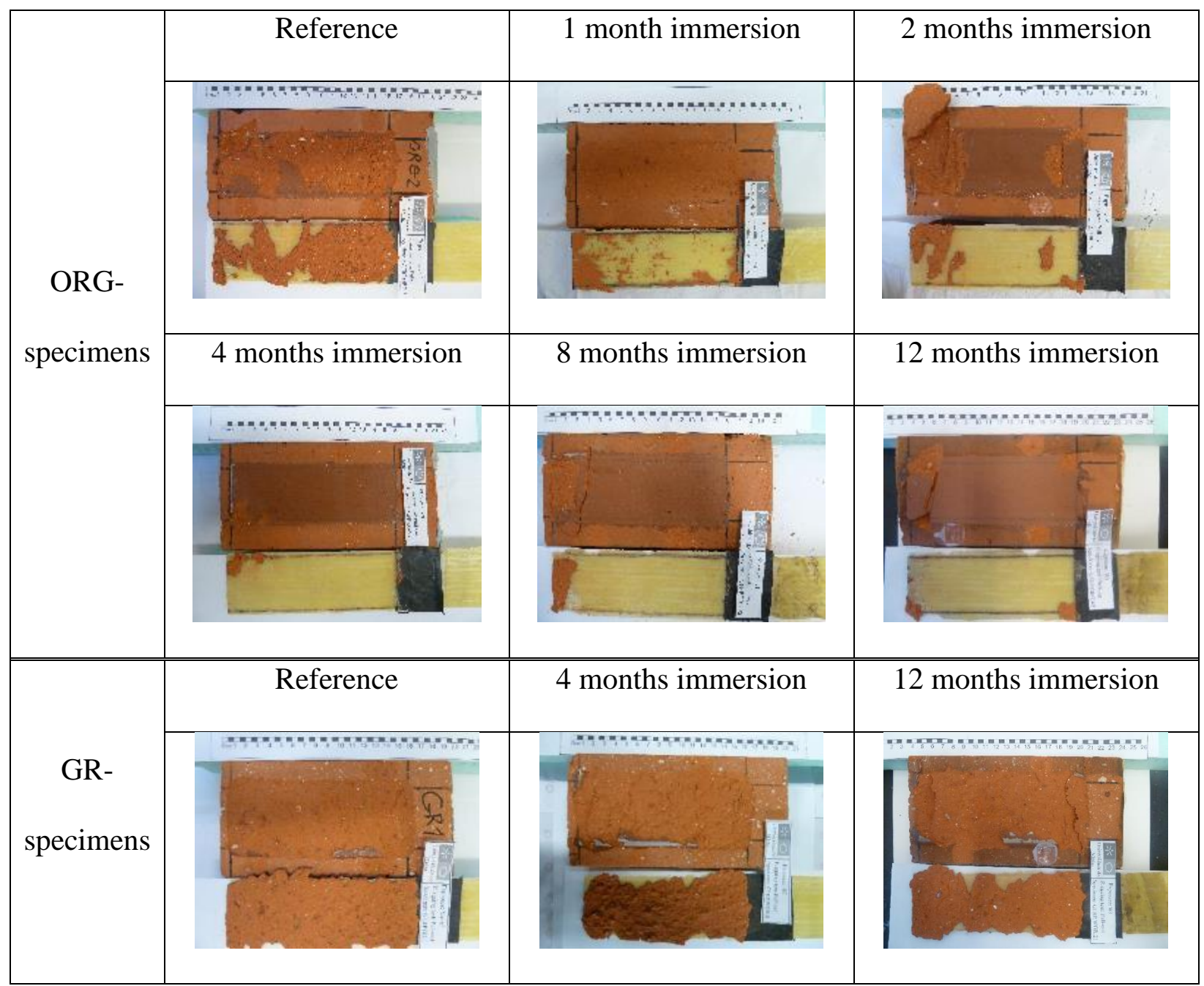

Fig 15. Failure mode changes with water immersion. 
(a)

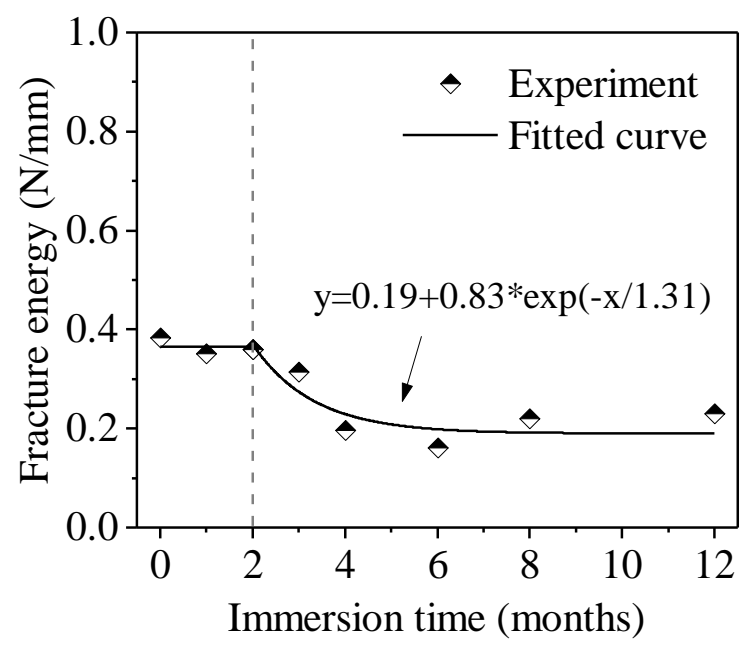

(b)

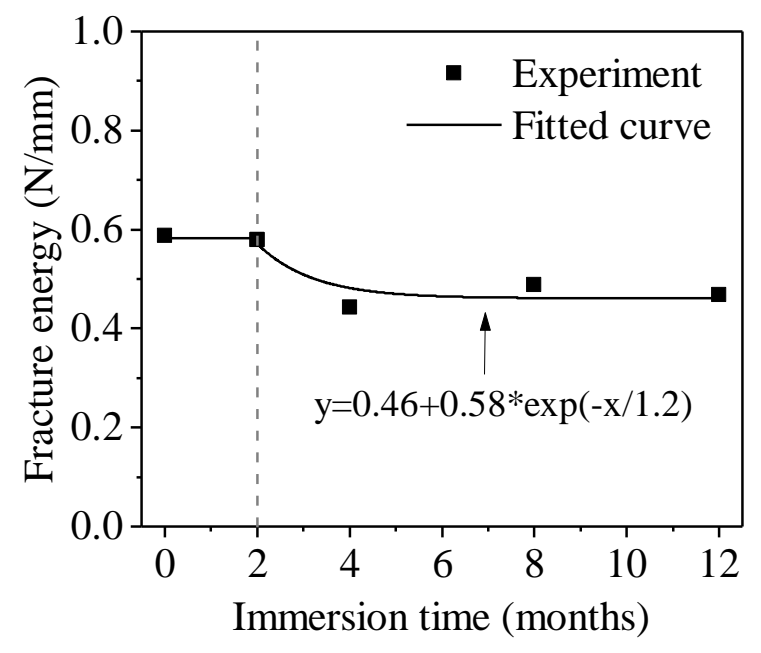

Fig 16. Bond fracture energy with immersion time in (a) ORG-specimens; (b) GR-specimens. 


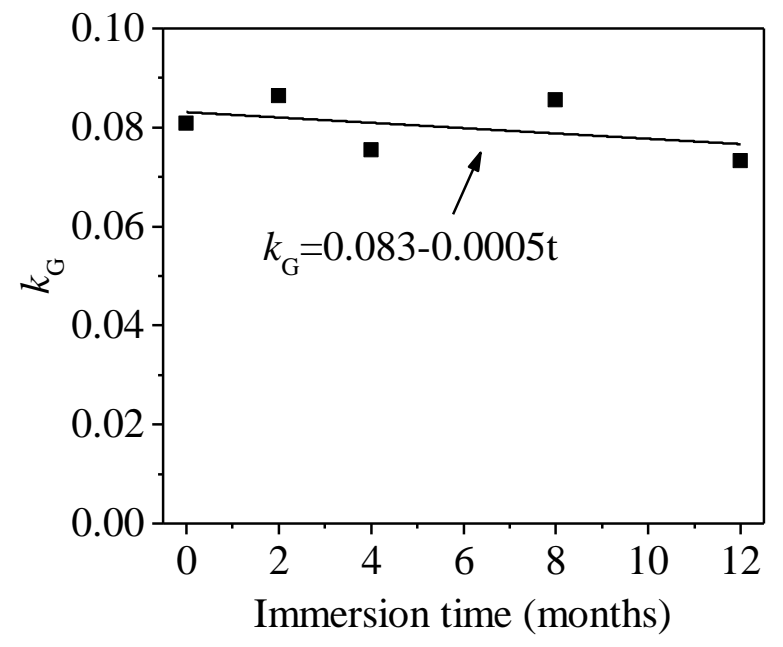

Fig 17. Changes of parameter $k_{\mathrm{G}}$ with immersion time. 